\title{
Partial splenic embolization combined with endoscopic therapies and NSBB decreases the variceal rebleeding rate in cirrhosis patients with hypersplenism: a multicenter randomized controlled trial
}

\author{
Xin Sun ${ }^{1}$ - Anzhong Zhang ${ }^{2} \cdot$ Tao Zhou $^{1} \cdot$ Minghui Wang $^{3} \cdot$ Yong Chen ${ }^{2} \cdot$ Ting Zhou $^{1} \cdot$ Xiaoning Chen $^{1} \cdot$ Aiyuan Xiu $^{1}$.

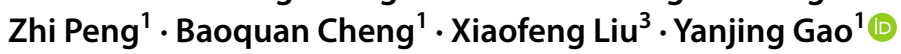

Received: 12 October 2020 / Accepted: 30 January 2021 / Published online: 27 February 2021

(c) The Author(s) 2021

\begin{abstract}
Background Global research on endoscopic therapies in combination with partial splenic embolization (PSE) for variceal hemorrhage (VH) is limited. Therefore, we aimed to evaluate the efficacy and safety of endoscopy plus PSE (EP) treatment in comparison to endoscopic (E) treatment for the secondary prophylaxis of VH in cirrhosis patients with hypersplenism. Methods Cirrhosis patients with hypersplenism (platelet count $<100,000 / \mu \mathrm{L}$ ) and those who had recovered from an episode of $\mathrm{VH}$ were enrolled in a multicenter randomized controlled trial. The participants were randomly assigned into EP and $\mathrm{E}$ groups in a 1:1 ratio. The primary endpoint was variceal rebleeding, and the secondary endpoints were severe variceal recurrence and mortality during the 2-year follow-up. Hematological indices, serum biochemical parameters, and the Child-Pugh score were measured at each time point.

Results From June 2016 to December 2019, 108 patients were enrolled in the study, among which 102 patients completed the protocol (51 in EP and 51 in E group). The rebleeding rate of the varices was significantly reduced in the EP group compared to that in the E group during the 2 years $(16 \%$ vs. $31 \%, p<0.001)$. The EP group showed a significantly lower variceal recurrence rate than the E group ( $22 \%$ vs. $67 \%, p<0.001)$. The COX proportional hazard models revealed that grouping was an independent predictor for variceal rebleeding $(H=0.122,95 \% \mathrm{CI} 0.055-0.270, p<0.001)$ and variceal recurrence (hazard ratio, $H=0.160,95 \%$ CI $0.077-0.332, p<0.001$ ). The peripheral blood cell count, Child-Pugh class/score, albumin concentration, and coagulation function in the EP group improved significantly compared to the values observed in the $\mathrm{E}$ group at any time point $(p<0.05)$.

Conclusions The EP treatment was more effective in preventing variceal rebleeding and variceal recurrence than the conventional $\mathrm{E}$ treatment during the secondary prophylaxis of $\mathrm{VH}$ in cirrhosis patients with hypersplenism. Furthermore, the EP treatment could significantly increase the peripheral blood cell count and albumin concentration and also improved the coagulation function and the Child-Pugh score.

Clinical trials registration Trial registration number ClincialTrials.gov: NCT02778425. The URL of the clinical trial: https ://clinicaltrials.gov/
\end{abstract}

Keywords Liver cirrhosis · Partial splenic embolization · Hypersplenism · Thrombocytopenia · Portal hypertension · Gastroesophageal varices $\cdot$ Secondary prophylaxis $\cdot$ Endoscopic variceal ligation $\cdot$ Non-selective beta-blocker . Cyanoacrylate injection

Xiaofeng Liu and Yanjing Gao Share correspondence authorship.

Xiaofeng Liu

liuxf0531@126.com

Yanjing Gao

gaoyanjing@sdu.edu.cn

1 Department of Gastroenterology, Qilu Hospital of Shandong University, Wenhua Xi Road, 107, Jinan City 250012,

Shandong Province, People's Republic of China
Department of Gastroenterology, Shandong Provincial Hospital Affiliated to Shandong University, Jinan City 250021, Shandong Province, China

3 Department of Gastroenterology, The 960th Hospital of the PLA Joint Logistics Support Force, Shifan Road, 25, Jinan City 250031, Shandong Province, People's Republic of China 


\section{Abbreviations \\ EV Esophageal varices \\ EVL Endoscopic variceal ligation \\ GEVs Gastroesophageal varices \\ GV Gastric varices \\ NSBB Non-selective beta-blocker \\ PSE Partial splenic embolization \\ VH Variceal hemorrhage}

\section{Introduction}

Varices and variceal hemorrhage $(\mathrm{VH})$ are the major complications of portal hypertension that cause high mortality in cirrhosis patients. Nearly half of the newly diagnosed patients with compensated cirrhosis develop gastroesophageal varices (GEVs) [1]. The annual risk of the first VH is approximately $5-15 \%$ and entails 6 -week mortality of 10-20\% [2]. The 1-year risk of rebleeding after the first episode of bleeding is $60 \%$, and the mortality rate is up to $33 \%$ $[3,4]$. Hence, secondary prophylaxis is necessary to prevent rebleeding in cirrhosis patients who had recovered from an episode of acute $\mathrm{VH}$.

According to the Baveno VI recommendations, nonselective beta-blocker (NSBB) combined with endoscopic variceal ligation (EVL) is recommended for the secondary prophylaxis of esophageal variceal hemorrhage. Cyanoacrylate injection in the patients with large GEV type 2 or isolated gastric varices $(\mathrm{GV})$ type 1 is promising for the prevention of first variceal bleeding [5]. A retrospective cohort study demonstrated the efficacy of continued EVL plus cyanoacrylate injection for the secondary prophylaxis of variceal bleeding in patients with concomitant esophageal varices (EV) and GV [6]. However, the application of cyanoacrylate in these patients should be investigated further with the evaluation of the risk/benefit ratio.

The incidence of hypersplenism in cirrhosis patients with portal hypertension is $11 \%-55 \%$ [7]. Hypersplenism is a major contributing factor in the development of thrombocytopenia, anemia, and leukopenia in such patients. Hypersplenism often develops in parallel with splenomegaly. Clinically, splenomegaly in cirrhosis patients is associated with a poor prognosis along with the maintenance of portal hypertension due to increased splenic blood flow. Moreover, thrombocytopenia and splenomegaly are independent predictors of bleeding from large varices [8]. Partial splenic embolization (PSE) is effective in improving the hematologic parameters, reducing the episodes of variceal bleeding, maintaining long-term eradication of the varices, and enhancing hepatic synthesis [9-11]. Unfortunately, there is no universally accepted therapy for GEVs in cirrhosis patients with hypersplenism, especially those with thrombocytopenia.
However, on the theoretical basis mentioned above, we consider that a combined therapy involving endoscopic therapies and PSE for GEVs in cirrhosis patients with hypersplenism would be sufficient. Therefore, we conducted a prospective study to assess the efficacy of EVL and cyanoacrylate injection combined PSE (EP treatment) vs. EVL and cyanoacrylate injection (E treatment) alone for the secondary prophylaxis of VH in cirrhosis patients with hypersplenism.

\section{Methods}

\section{Study design and patients}

The present study was a prospective, multicenter, doubleblind, randomized controlled trial. The study was carried out at the Gastroenterology Department of three tertiary referral hospitals (Qilu Hospital of Shandong University, the 960th Hospital of the PLA Joint Logistics Support Force, and Shandong Provincial Hospital Affiliated to Shandong University) that treat most of the patients with liver cirrhosis in China. The study was approved by the Ethics Committee of Shandong University. Each institution approved the protocol, and the data of all patients were recorded in a standard form. All patients were informed of the aims of the study and signed the informed consent before participation. This study was registered at ClinicalTrials.gov with the identifier number: NCT02778425.

The enrollment criteria were: (i) patients aged between 18 and 75 years; (ii) patients who had recovered from an episode of $\mathrm{VH}$ or patients who had survived from acute $\mathrm{VH}$ and there was no bleeding for consecutive 5 days; (iii) patients with a diagnosis of liver cirrhosis and portal hypertension on clinical examination, laboratory test, and imaging or histological examination; and (iv) patients with hypersplenism and thrombocytopenia (platelets $<100,000 / \mu \mathrm{L}$ ).

The exclusion criteria were: (i) previous therapy (splenectomy, PSE, EVL, tissue adhesive injection, or usage of NSBB) to prevent rebleeding; (ii) bleeding from isolated gastric or ectopic varices; (iii) hepatocellular carcinoma or other malignant tumors; (iv) contraindications for the use of NSBBs, hepatic failure, and Child-Pugh class $\mathrm{C}$ with large amount ascites, or grade 3-5 hepatic encephalopathy, or prothrombin activity $\leq 40 \%$; (v) hepatic failure; (vi) contraindications for PSE; (vii) pregnancy and lactation; and (viii) inability to sign the informed consent.

\section{Randomization and masking}

The patients qualifying the inclusion criteria for this study were randomly assigned into EP and E groups in a 1:1 ratio. Randomization was carried out according to a 
computer-generated randomization sequence. Patient allocation and assignment were performed using a sealed opaque envelope. The endoscopist, interventional radiologist, and trial statistician were blinded to the treatment assignments.

\section{Endoscopic treatment protocols}

On the premise of hemodynamic stability, the patients underwent endoscopic examination and endoscopic treatment after receiving intravenous anesthesia under the supervision of an anesthesiologist. All the diagnostic and therapeutic endoscopies were performed using PENTAX Gastroscopy (EG29-i10, PENTAX) by a technically proficient gastrointestinal endoscopist and the support staff. During the first session, cyanoacrylate (Histoacryl glue, Braun, Melsungen, Germany, or Beijing Component Medical Devices Co. Ltd.) was injected using a 23-gauge disposable injection needle (NM-200L-0521, Olympus, Tokyo, Japan) into the GV following the standardized sandwich method (normal saline + cyanoacrylate + normal saline). Each shot contained no more than $2.0 \mathrm{~mL}$ cyanoacrylate plus an equal volume of normal saline. In each session, no more than six injections were administered. Furthermore, EVL was carried out with a 6 Shooter Saeed Multi-Band Ligator (Cook Endoscopy, Inc., Winston-Salem, NC, USA) at $1 \mathrm{~cm}$ above the Z-line in a spirally ascending fashion.

After the initial session, EVL was performed at regular intervals of about 4 weeks. If necessary, additional cyanoacrylate was injected into distinct large GV until the varices were eradicated.

\section{PSE protocols}

PSE was performed 1 week before the endoscopic treatment in the EP group. All patients underwent enhanced computed tomography (CT) scanning to observe the basal status of the spleen. Initially, splenic arteriography was performed on a digital subtraction angiography (DSA) system via the femoral artery access. Using the Seldinger technique to apply a 5 French guiding catheter (Terumo, Tokyo, Japan) into the femoral artery, the contrast agent was administered to demonstrate the splenic artery and the collateral circulation routes. A microcatheter was located distally in the splenic hilus by superselective catheterization. Progressive embolization was performed by repeated injections of a gelatin sponge cut into 1-2-mm cubes under the DSA control until a $60-80 \%$ reduction in the splenic blood flow was achieved.

Further observation and management for all the patients were performed in the hospital. Broad-spectrum antibiotic prophylaxis was administered in the perioperative period.
A post-interventional enhanced CT scan was performed to confirm the embolization area 2-3 weeks after the PSE.

\section{Additional treatment}

A standard dose of NSBB (propranolol) was applied to patients according to the Baveno VI recommendations if there were no contraindications. The dosage of propranolol was titrated to either reduce the resting heart rate by $25 \%$ or achieve a heart rate of up to 55 beats /min, or a maximum tolerated dose was used. All the post-hepatitis B cirrhotic participants received anti-viral therapy.

\section{Outcomes and definitions}

The primary endpoint of the study was variceal rebleeding during the 2-year follow-up. The secondary endpoints were severe variceal recurrence and mortality. Hematological indices, serum biochemical parameters, and the Child-Pugh score were measured at each time point.

Eradication of the varices was defined as no visible varices in endoscopy or the presence of only fibrosed varices not requiring ligation. Variceal bleeding was defined as active hematemesis and/or melena, presence of active esophageal or esophagogastric variceal bleeding in endoscopy, red signs of varix, or with plug overlying varix. Recurrence of varices referred to the detection of new varices after variceal eradication had been previously achieved.

\section{Follow-up}

After variceal eradication was achieved, a routine followup was performed for each patient at the time points of 3 months, 6 months, 1 year, and 2 years. The follow-up visit included clinical assessment, endoscopic follow-up, evaluation of hematological parameters, serum biochemical analysis, and Child-Pugh score. Follow-up endoscopy was performed every 3 months, and if there was no recurrence, after every 6 months. Once the patients suffered from variceal bleeding or generated new varices with forms F2/F3 [12] as observed in endoscopy, they were dropped out of the group.

\section{Statistical analysis}

Statistical analysis was performed using the Statistical Program for Social Science (SPSS) version 11.0 for Windows (SPSS, Chicago, IL). The sample size was estimated based on the varices rebleeding rate in the secondary prophylaxis. Assuming the rebleeding rate to be $37 \%$ in the E group and $15 \%$ in the EP group, and 5\% $\alpha$ and $10 \% \beta$ error, respectively, approximately 110 patients were included considering a $20 \%$ dropout rate. 
The continuous variables were expressed as mean \pm standard deviation (SD) or (interquartile range)/M (IQR) and analyzed by two-sample Student's $t$ tests or Mann-Whitney $U$ test for comparison. The categorical variables were expressed as frequency and percentage and were analyzed using the Chi-squared test.

The rebleeding and recurrence rates of the varices were analyzed by the Kaplan-Meier survival curve. The log-rank test was used for a comparison between the two groups. The Cox univariate analysis was performed to assess the candidate variables significantly $(p<0.20)$. Next, the candidate variables, along with the other variables that were thought to be necessary for contributions, were included in the Cox multivariate analysis to determine the independent factors predicting variceal rebleeding and variceal recurrence. A $p$-value of less than 0.05 was considered statistically significant.

\section{Results}

\section{Characteristics and follow-up of the patients}

From June 2016 to December 2019, 108 patients were enrolled in the randomized trial and divided into two groups in a 1:1 ratio. Two patients from the EP group and three from the E group were discontinued from the endoscopic treatment because of poor compliance. One patient from the EP group died due to acute variceal bleeding induced by discontinued endoscopic treatment and hard food consumption 2 months after the PSE. Finally, 51 patients from the EP group and 51 patients from the E group completed the per-protocol analysis (Fig. 1).

The baseline characteristics shown in Table 1 were well-matched $(p>0.05)$ in the two groups. Two patients

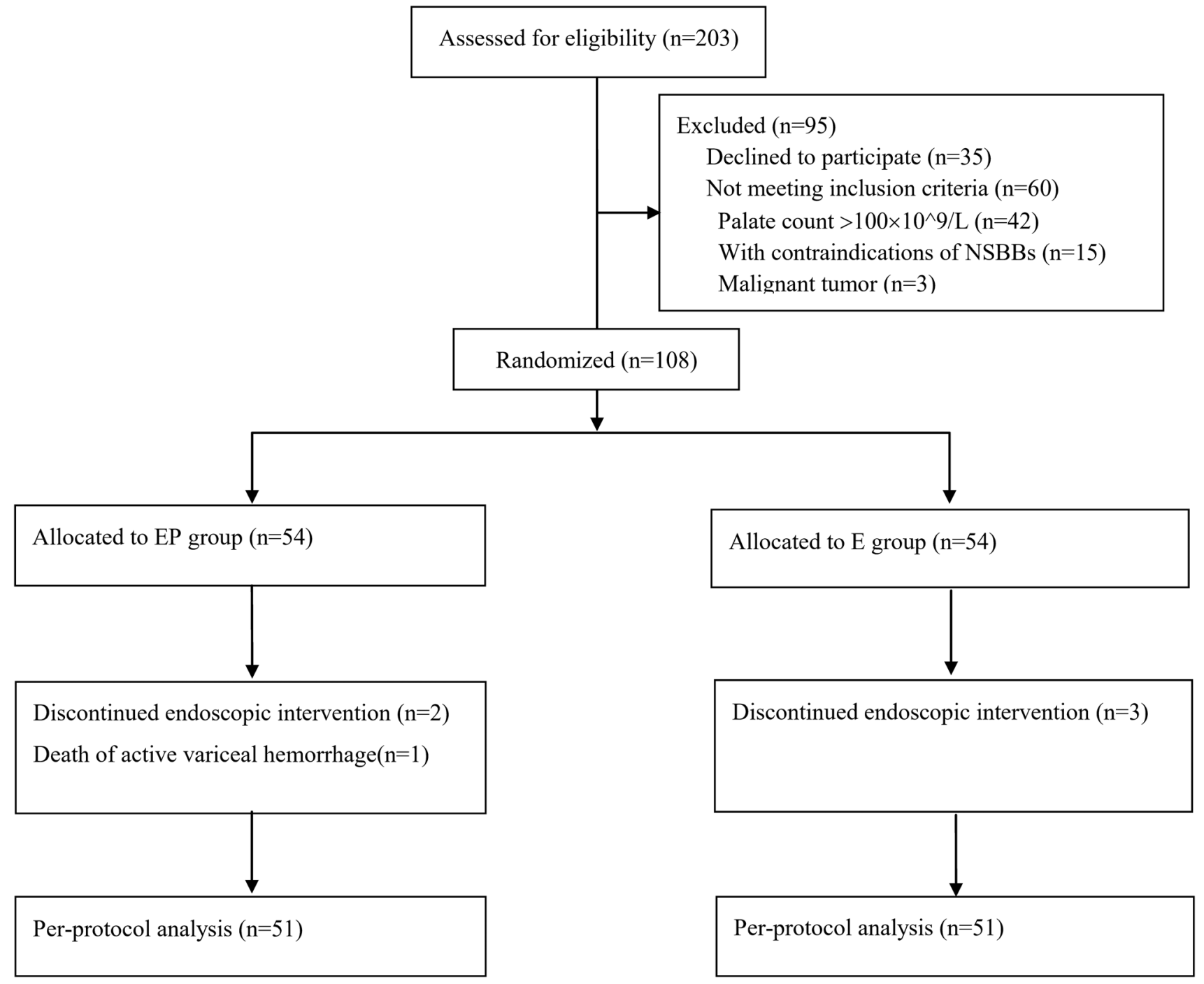

Fig. 1 Flow diagram of the study. PSE Partial splenic embolization 
from the EP group and three patients from the E group were discontinued from the NSBB treatment due to poor compliance $(p>0.05)$.

\section{Variceal rebleeding}

After the initial endoscopic or EP treatment, variceal rebleeding occurred in a total of 24 patients, including eight patients in the EP group and 16 patients in the $\mathrm{E}$ group $(16 \%$ vs. $31 \%, \log$-rank $p=0.008)$. The Kaplan-Meier curve for rebleeding is presented in Fig. 2a. The median variceal rebleeding period was 41 months in the E group. According to the COX proportional hazard models, the only independent factor predicting the risk of variceal rebleeding was treatment with EP (hazard ratio $=0.122,95 \%$ CI $0.055-0.270, p=0.000)($ Table 2).

\section{Variceal recurrence}

During the follow-up, 11 patients (22\%) in the EP group and 34 patients $(67 \%)$ in the E group generated new varices that required further endoscopic prophylactic therapy. As demonstrated by the Kaplan-Meier analysis (Fig. 2b), the recurrence rate in the EP group was significantly lower than that in the E group (log-rank $p<0.001)$. Only one patient in the EP group and two in the E group generated new varices with form F2. The median variceal recurrence period was 11 months in the E group. The COX proportional hazard models revealed that treatment with EP (hazard ratio $=0.160,95 \%$ CI $0.077-0.332$, $p=0.000$ ) was the only independent factor predicting the risk of variceal recurrence (Table 3 ).

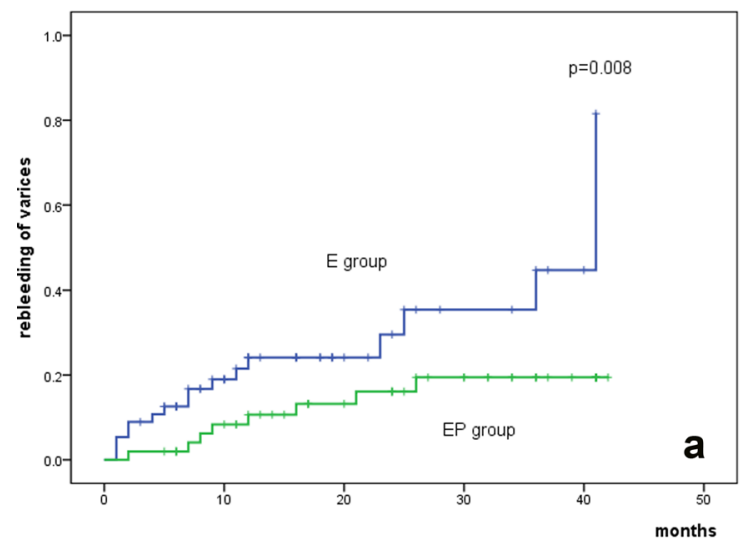

\section{Chronological changes in the peripheral blood cell count}

The white blood cell (WBC) count, red blood cell (RBC) count, platelet (PLT) count, and hemoglobin (HGB) level in the EP group were significantly increased compared to those in the $\mathrm{E}$ group at any time point during the 2-year follow-up $(p<0.05$; Fig. 3a-d).

The subgroup analysis revealed significant improvements in the peripheral blood cell count in the EP group during the follow-up. First, the WBC count increased significantly after the treatment and this increase was maintained for 2 years $(p<0.05$; Fig. $3 a)$. The WBC count increased from 27 to $79 \%$ during the follow-up compared to the baseline value. Simultaneously, the median RBC and the hemoglobin (HGB) level displayed a significant increment from 20 to $35 \%$ and from 25 to $40 \%$, respectively, compared to the baseline value; these increments were maintained for 2 years $(p<0.05$; Fig. $3 \mathrm{~b}, \mathrm{~d})$. In addition, the PLT count almost doubled from $61.08 \pm 19.6 \times 10^{9} / \mathrm{L}$ to a mean value of $123.76 \pm 64.71 \times 10^{9} / \mathrm{L}$ after the treatment. Although the PLT count decreased over time, it remained significantly elevated in comparison to the pre-procedural levels $(p<0.05$; Fig. 3c). As for the E group, the WBC count, RBC count and HGB level showed no significant post-procedural difference at any time point. Moreover, the PLT count in the E group decreased significantly 1 year after the procedure $(p<0.05$; Fig. 3c).

\section{Chronological changes in the liver function parameters}

The albumin (ALB) concentration in the EP group increased significantly compared to that in the E group at any time point after treatment. In the E group, the ALB concentration decreased significantly 1 year after the procedure $(p<0.05$;

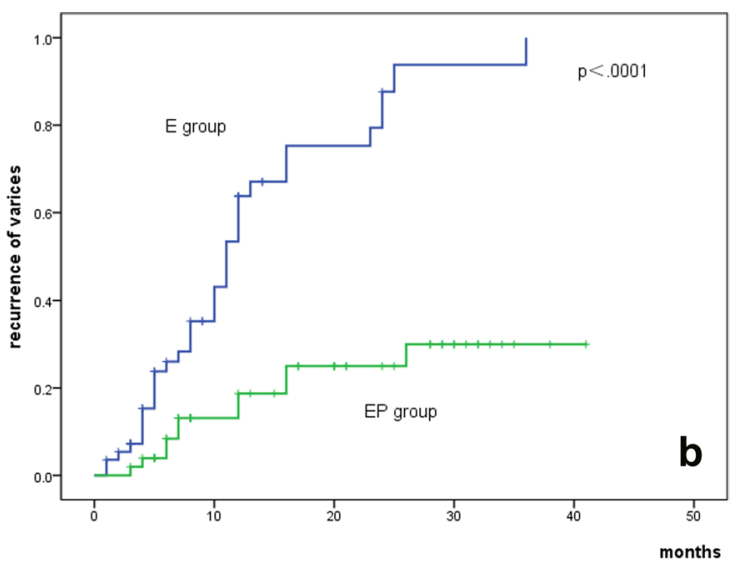

Fig.2 Kaplan-Meier curves of variceal rebleeding (a), variceal recurrence (b). The log-rank $p$ values of which were 0.000 and 0.008 , respectively; PSE partial splenic embolization 
Table 1 Baseline characteristics of the patients

\begin{tabular}{|c|c|c|c|}
\hline & EP group $(n=51)$ & E group $(n=51)$ & $p$ value \\
\hline Age (years) & $52.26 \pm 9.93$ & $54.69 \pm 9.73$ & $0.22^{\mathrm{a}}$ \\
\hline \multicolumn{4}{|l|}{ Gender, $n(\%)$} \\
\hline Male & $27(53 \%)$ & $36(71 \%)$ & \multirow[t]{2}{*}{$0.10^{\mathrm{c}}$} \\
\hline Female & $24(47 \%)$ & $15(29 \%)$ & \\
\hline \multicolumn{4}{|c|}{ Etiology of cirrhosis, $n(\%)$} \\
\hline Hepatitis B virus & $28(55 \%)$ & $27(54 \%)$ & \\
\hline Hepatitis $\mathrm{C}$ virus & $3(6 \%)$ & $4(7 \%)$ & \\
\hline $\begin{array}{l}\text { Alcoholic liver } \\
\text { disease }\end{array}$ & $3(6 \%)$ & $5(12 \%)$ & \multirow[t]{3}{*}{$0.68^{\mathrm{c}}$} \\
\hline $\begin{array}{l}\text { Autoimmune hepa- } \\
\text { titis }\end{array}$ & $1(2 \%)$ & $5(9 \%)$ & \\
\hline Unexplained cause & $16(31 \%)$ & $10(18 \%)$ & \\
\hline $\begin{array}{l}\text { Child-Pugh score, } \\
n(\%)\end{array}$ & $6.63 \pm 1.20$ & $6.77 \pm 1.70$ & \multirow[t]{2}{*}{$0.83^{\mathrm{b}}$} \\
\hline Class A & $29(57 \%)$ & $29(57 \%)$ & \\
\hline Class B & $21(41 \%)$ & $19(37 \%)$ & \multirow[t]{2}{*}{$0.92^{\mathrm{b}}$} \\
\hline Class $\mathrm{C}$ & $1(2 \%)$ & $3(6 \%)$ & \\
\hline \multicolumn{4}{|c|}{ Degree of esophageal varices, $n(\%)$} \\
\hline $\mathrm{F} 1$ & $1(2 \%)$ & $1(2 \%)$ & \\
\hline $\mathrm{F} 2$ & $11(22 \%)$ & $5(10 \%)$ & \multirow[t]{2}{*}{$0.13^{\mathrm{b}}$} \\
\hline F3 & $39(76 \%)$ & $45(88 \%)$ & \\
\hline $\begin{array}{l}\text { White blood cell } \\
\left(\times 10^{9} / \mathrm{L}\right)\end{array}$ & $2.73 \pm 1.29$ & $2.77 \pm 1.47$ & $0.89^{\mathrm{a}}$ \\
\hline $\begin{array}{l}\text { Red blood cell } \\
\left(\times 10^{12} / \mathrm{L}\right)\end{array}$ & $3.21 \pm 0.74$ & $3.28 \pm 0.81$ & $0.66^{\mathrm{a}}$ \\
\hline Platelet $\left(\times 10^{9} / \mathrm{L}\right)$ & $61.08 \pm 19.53$ & $66.45 \pm 18.26$ & $0.15^{\mathrm{a}}$ \\
\hline Hemoglobin $(\mathrm{g} / \mathrm{L})$ & $86.18 \pm 26.36$ & $89.90 \pm 26.02$ & $0.47^{\mathrm{a}}$ \\
\hline Prothrombin time (s) & $15.11 \pm 2.41$ & $14.76 \pm 1.95$ & $0.43^{\mathrm{a}}$ \\
\hline INR & $1.33 \pm 0.21$ & $1.29 \pm 0.17$ & $0.31^{\mathrm{a}}$ \\
\hline $\begin{array}{l}\text { Prothrombin activity } \\
(\%)\end{array}$ & $65.08 \pm 13.22$ & $66.31 \pm 13.55$ & $0.64^{\mathrm{a}}$ \\
\hline ALT(U/L) & $24.57 \pm 18.71$ & $40.80 \pm 103.06$ & $0.22^{\mathrm{a}}$ \\
\hline AST(U/L) & $29.71 \pm 16.62$ & $51.94 \pm 124.14$ & $0.21^{\mathrm{a}}$ \\
\hline $\begin{array}{l}\text { Alkaline phosphatase } \\
\text { (U/L) }\end{array}$ & $80.14 \pm 35.21$ & $96.88 \pm 58.94$ & $0.08^{\mathrm{a}}$ \\
\hline Albumin $(\mathrm{g} / \mathrm{L})$ & $36.43 \pm 5.91$ & $34.92 \pm 6.55$ & $0.22^{\mathrm{a}}$ \\
\hline $\begin{array}{l}\text { Total bilirubin } \\
\quad(\text { umol/L) }\end{array}$ & $21.04 \pm 15.85$ & $20.68 \pm 10.68$ & $0.89^{\mathrm{a}}$ \\
\hline $\begin{array}{l}\text { Follow-up duration } \\
\text { (months) }\end{array}$ & $21.53 \pm 3.14$ & $20.87 \pm 2.15$ & $0.24^{\mathrm{a}}$ \\
\hline
\end{tabular}

Data are means \pm standard deviations

$I N R$ international normalized ratio. $A L T$ alanine aminotransferase, AST:aspartate aminotransferase.

${ }^{\text {a }}$ Determined with the $t$ test

${ }^{\mathrm{b}}$ Determined with Mann-Whitney $U$ test

${ }^{\mathrm{C}}$ Determined with the Chi-squared test

Fig. 4). The levels of alanine aminotransferase level (ALT), aspartate aminotransferase (AST), alkaline phosphatase (AKP), and total bilirubin exhibited no significant difference between the two groups at any time point.

\section{Chronological changes in the coagulation function}

The coagulation function parameters, such as the prothrombin time (PT) (Fig. 5a), international normalized ratio (INR) (Fig. 5b), and prothrombin activity (PTA) (Fig. 5c), in the EP group showed significant improvement after the treatment when compared to those in the E group. Moreover, the EP treatment significantly improved the coagulation function parameters at any time point during the follow-up $(p<0.05$; Fig. 5). The median INR value decreased significantly over time from 1.33 before PSE to 1.20 over 2 years. The median PT also showed a significant decrease from $15.11 \mathrm{~s}$ before PSE to $13.40 \mathrm{~s}$ over 2 years. The value of PTA in the EP group gradually increased by $22 \%$ compared to the baseline.

\section{Child-Pugh class/score}

The Child-Pugh class/score in the EP group exhibited significant improvement compared to the baseline and this improvement persisted for 2 years. Indeed, the EP group presented a significantly reduced Child-Pugh score 6 months after the treatment compared to the E group (Table 4).

\section{Changes in the portal vein diameter and times of endoscopic treatment}

The diameters of the main portal vein (MPV) measured using CT angiography did not exhibit any significant difference $(p>0.05)$ before PSE $(20.41 \pm 4.14 \mathrm{~mm})$ and after PSE $(18.81 \pm 3.44 \mathrm{~mm})$. The times of EVL in the EP group $(1.98 \pm 0.81)$ were significantly reduced compared to those in the E group (3.65 \pm 0.10$)$. Moreover, the bands assumption in the EP group $(14.57 \pm 7.47)$ were significantly lower than those in the E group $(25.59 \pm 8.49)$. The times of cyanoacrylate injection in the EP group $(1.31 \pm 0.62)$ were significantly reduced than those in the E group $(1.82 \pm 1.01)$. Furthermore, the glue assumption in the EP group $(2.06 \pm 2.15)$ was significantly reduced than that in the E group $(3.93 \pm 4.02)$.

\section{Adverse events}

After the endoscopic treatment, about one-third of the patients experienced endoscopy-related adverse events such as retrosternal pain (28\%), nausea (12\%), moderate fever (below $39.0{ }^{\circ} \mathrm{C} ; 4 \%$ ), which usually palliated spontaneously after 2-3 days without any special management. Two patients in the E group experienced postband ligation hemorrhage as detected in endoscopy. The hemorrhage was controlled pharmacologically after hospitalization. After the PSE procedure, the majority of the patients exhibited at least one symptom of the post-embolic syndrome, such as abdominal pain (74\%), 
Table 2 Factors predicting variceal rebleeding

\begin{tabular}{llll}
\hline Variables & $\begin{array}{l}\text { COX univariate analysis } \\
(p \text { value })\end{array}$ & $\begin{array}{l}\text { COX multivariate analy- } \\
\text { sis }(p \text { value })\end{array}$ & Hazard ratio(95\% CI $)$ \\
\hline Age & 0.710 & - & - \\
Gender & 0.107 & - & - \\
Etiology & 0.773 & 0.795 & - \\
Child-Pugh score & 0.261 & 0.695 & - \\
White blood cell & 0.664 & - & - \\
Red blood cell & 0.400 & - & - \\
Platelet & 0.670 & 0.197 & - \\
Prothrombin time & 0.849 & 0.062 & - \\
INR & 0.699 & 0.076 & - \\
Prothrombin activity & 0.938 & - & - \\
ALT & 0.001 & 0.822 & - \\
AST & 0.002 & 0.802 & - \\
Albumin & 0.836 & 0.880 & $0.122(0.055-0.270)$ \\
Total bilirubin & 0.341 & - &
\end{tabular}

$C I$ confidence interval, INR international normalized ratio, ALT alanine aminotrans-ferase, AST: aspartate aminotransferase

\begin{tabular}{llll}
\hline Variables & $\begin{array}{l}\text { COX univariate analysis } \\
(p \text { value })\end{array}$ & $\begin{array}{l}\text { COX multivariate analy- } \\
\text { sis }(p \text { value })\end{array}$ & Hazard ratio (95\% CI) \\
\hline Age & 0.747 & & - \\
Gender & 0.145 & & - \\
Etiology & 0.988 & 0.235 & - \\
Child-Pugh score & 0.218 & & - \\
White blood cell & 0.934 & 0.092 & - \\
Red blood cell & 0.323 & 0.603 & - \\
Platelet & 0.422 & 0.213 & - \\
Prothrombin time & 0.932 & 0.967 & - \\
INR & 0.706 & & - \\
Prothrombin activity & 0.950 & & - \\
ALT & 0.178 & 0.694 & - \\
AST & 0.202 & & - \\
Albumin & 0.746 & 0.000 & 0.160 (0.077-0.332) \\
Total bilirubin & 0.326 & & \\
Treatment & 0.003 &
\end{tabular}

$C I$ confidence interval, INR international normalized ratio, $A L T$ alanine aminotrans-ferase;, $A S T$ aspartate aminotransferase
Table 3 Factors predicting variceal recurrence abdominal distention (56\%), nausea (7\%), and fever (below $39.0{ }^{\circ} \mathrm{C} ; 65 \%$ ); the symptoms were managed medically prior to discharge. One patient with Child-Pugh Class B developed portal vein thrombosis that was observed during a CT angiography examination 3 weeks after PSE. This patient was treated with anticoagulant for 6 months, which completely recanalized the portal vein. No severe complications, such as splenic abscess, splenic rupture, serious infection, and sepsis, were observed post-PSE. Mortality related to the endoscopic therapy or the PSE procedure was not recorded during the follow-up.

\section{Discussion}

Liver cirrhosis patients, especially those with additional complications such as severe hypersplenism and thrombocytopenia and those who survive acute variceal bleeding, 
Fig.3 Follow-up results of peripheral blood cell count. a White blood cell (WBC), b red blood cell (RBC), c platelet (PLT), $\mathbf{d}$ hemoglobin (HGB) in EP group and $\mathrm{E}$ group. PSE partial splenic embolization. *There was a significant difference between the EP group and $\mathrm{E}$ group $(p<0.05)$. **It was significantly increased at this point than the pre-EP treatment $(p<0.05)$. *** It was significantly increased/decreased at this point than the pre-E therapy $(p<0.05)$
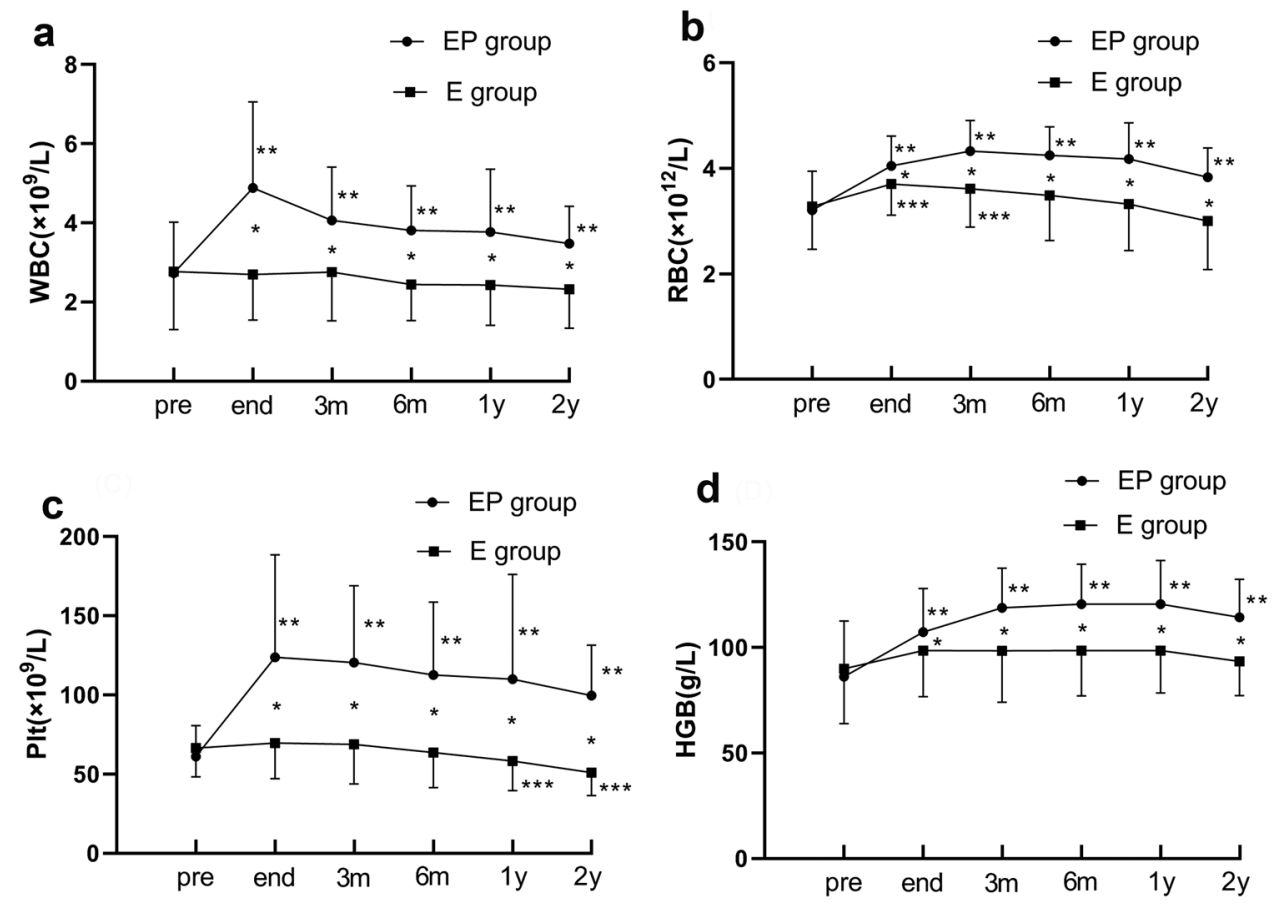

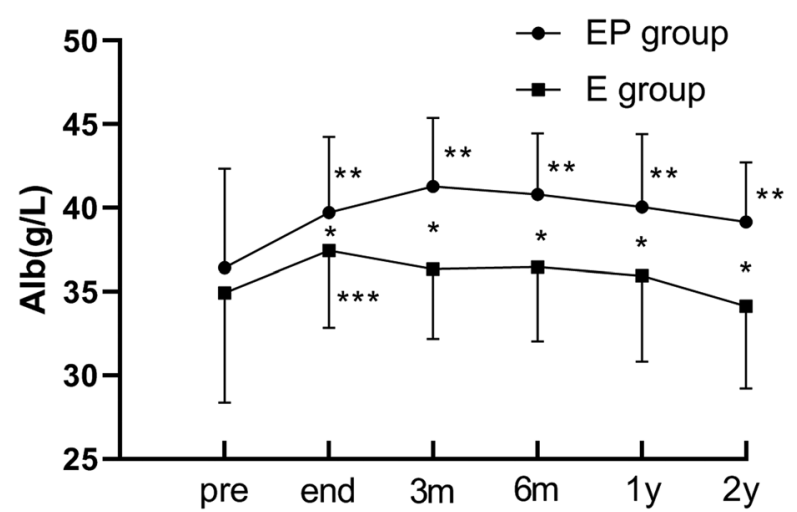

Fig.4 Follow-up results of albumin concentration. PSE, partial splenic embolization. *There was a significant difference between the EP group and $\mathrm{E}$ group $(p<0.05)$. **It was significantly increased at this point than the pre-EP treatment $(p<0.05)$. $* * *$ It was significantly increased at this point than the pre-E therapy $(p<0.05)$

exhibit a high rate of rebleeding and mortality. However, the optimal management of variceal hemorrhage in patients with hypersplenism is not well understood [5]. Therefore, we first attempted using the EP treatment for the management of variceal rebleeding in a multicenter randomized controlled trial. Our results showed that the EP treatment could significantly reduce the variceal rebleeding and recurrence rate compared to the $\mathrm{E}$ treatment. Moreover, the EP treatment resulted in a significant long-term improvement in the peripheral blood cell count, liver function and coagulation function.
Treatments such as endoscopy, portosystemic shunts, and pharmacotherapy are attempted to decrease the risk of rebleeding and mortality [13]. Currently, the first-line therapy for the secondary prophylaxis of esophageal variceal hemorrhage is EVL plus NSBB [3]. However, adopting only endoscopic therapy as a treatment to eradicate varices cannot fundamentally relieve portal hypertension nor can it reduce the risk of rebleeding. Varices often recur with a $50 \%$ rate at 2 years, since the portal pressure and blood flow remain unchanged [14]. Several researchers have demonstrated that the portal pressure is positively correlated with the spleen size and that portal pressure can induce splenomegaly. Splenomegaly and hypersplenism are common in patients with cirrhosis, both usually accompanying each other $[15,16]$. In cirrhosis patients with hypersplenism, the extremely low platelet count may promote rebleeding [17]. Regardless of these factors, the primary goal of variceal management should be the prevention of variceal rebleeding and recurrence after variceal eradication.

In a series of clinical trials, PSE alone or in combination with conventional therapies was reported to be beneficial in reducing the risk of variceal bleeding and variceal recurrence in cirrhosis patients [18-20]. However, no research has been conducted on the secondary prophylaxis management of gastroesophageal variceal hemorrhage in cirrhosis patients with hypersplenism. Our study showed that the EP treatment was significantly associated with a lower incidence of variceal bleeding and variceal recurrence. Moreover, group assignment emerged as the only predictor for variceal bleeding and variceal recurrence. It was observed that after 


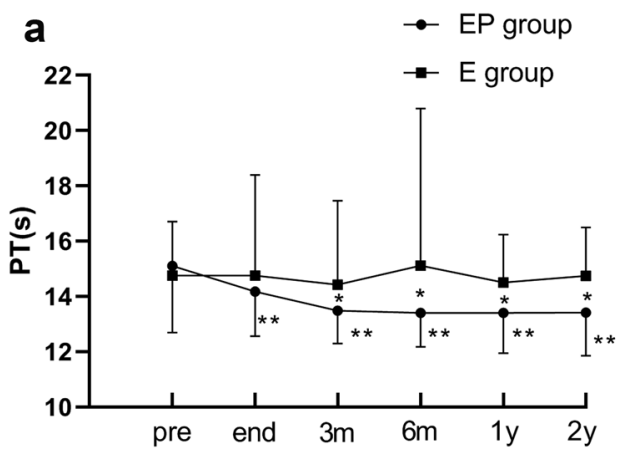

Fig.5 Follow-up results of coagulation function parameters. a prothrombin time (PT), b international normalized ratio (INR), c prothrombin activity (PTA) in EP group and E group. PSE partial

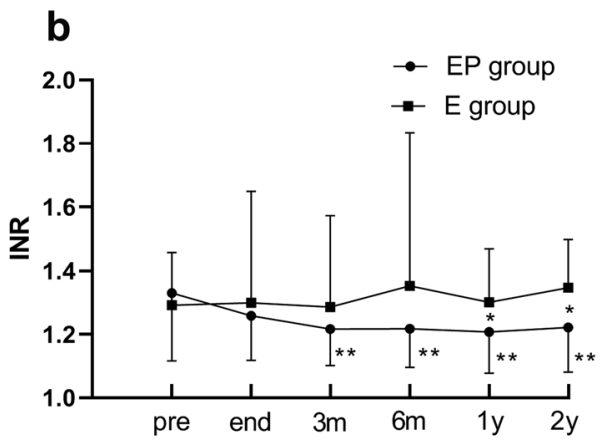

splenic embolization. *There was a significant difference between the EP group and E group $(p<0.05)$. **It was significantly increased/ decreased at this point than the pre-EP treatment $(p<0.05)$
Table 4 Follow-up results of Child-Pugh class/score

\begin{tabular}{|c|c|c|c|c|c|c|}
\hline \multirow[t]{2}{*}{ Time } & \multicolumn{2}{|l|}{ Child-Pugh class } & \multicolumn{4}{|c|}{ Child-Pugh score } \\
\hline & EP group $(A / B / C)$ & $\mathrm{E} \operatorname{group}(\mathrm{A} / \mathrm{B} / \mathrm{C})$ & $p$ & EP group & E group & $p$ \\
\hline Pre-treatment & $29 / 21 / 1$ & $29 / 19 / 3$ & 0.866 & $6.00(1)$ & $6.00(3)$ & 0.702 \\
\hline End of the treatment & $39 / 12 / 0 *$ & $34 / 16 / 1$ & 0.254 & $6.00(1)^{*}$ & $6.00(2)^{*}$ & 0.904 \\
\hline \multicolumn{7}{|l|}{ Post of the treatment } \\
\hline 3 months & $43 / 7 / 0^{*}$ & $38 / 11 / 2$ & 0.132 & $5.00(1)^{*}$ & $6.00(2)^{*}$ & 0.131 \\
\hline 6 months & $45 / 4 / 0^{*}$ & $36 / 15 / 0$ & 0.007 & $5.00(1)^{*}$ & $6.00(2)$ & 0.074 \\
\hline 1 year & $41 / 8 / 0^{*}$ & $27 / 16 / 2$ & 0.009 & $6.00(1)^{*}$ & $6.00(2)$ & 0.008 \\
\hline 2 years & $27 / 7 / 0^{*}$ & $15 / 13 / 3$ & 0.006 & $6.00(1)^{*}$ & $7.00(2)$ & 0.025 \\
\hline
\end{tabular}

Data are median (Interquartile Range)/M (IQR) or numbers of patients. Statistical analysis was performed using Mann-Whitney $U$ test

*It was significantly different at this time point than pre-treatment the EP treatment, the diameter of MPV had decreased, although the difference was not significant $(p>0.05)$. The procedure times of EVL, cyanoacrylate injection, and the assumption of bands and glue were significantly reduced in the EP group $(p<0.05)$. The reduction in time could be attributed to portal decompression after PSE, which unfortunately cannot be confirmed as we did not measure the portal vein pressure in this study. In the presence of collateral circulation, especially esophageal varices, both splenic and superior mesenteric arteries exhibit significantly high flow volumes. Around 60-80\% dearterialization occurs after PSE, and the splenic venous outflow and pressure decreases, following which the left and short gastric veins of the varices collapse [21]. Hence, after PSE, the frequency of endoscopic procedures decreases and management by endoscopic physicians become easier. PSE was reported to reduce the size of the spleen, splenic blood flow, splenic venous pressure, and portal venous pressure, and improve the hepatic arterial hypertensive state in cirrhotic patients, similar to the findings of the present study [22]. Furthermore, the improvement in portal hemodynamics is often associated with reduced bleeding from the esophageal or gastric varices.
Our results showed that the EP group reduced the variceal rebleeding rate by $48.4 \%$ and the variceal recurrence rate by $67.2 \%$ compared to the rates observed in the E group. Thus, the EP treatment is indeed better for the management of the secondary prophylaxis of $\mathrm{VH}$ and the reduction of variceal bleeding.

The current study indicated that both PLT and WBC counts were significantly increased within 2 weeks of PSE. Moreover, the cirrhosis patients with more than $50 \%$ splenic infarction presented a long-term improvement in leukocytopenia and thrombocytopenia, although the peripheral blood cell count declined consistently over time [23-25]. In our study, we demonstrated again that the WBC and PLT counts increased significantly within weeks of undergoing PSE. However, the counts decreased consistently in the subsequent months and years, although they were significantly better than those at the baseline. Moreover, the RBC count and the HGB level were also significantly elevated in the two groups, especially after the EP treatment.

The immediate improvement in the WBC count due to PSE could be attributed to the release of white cells from the spleen, considering it as an acute inflammatory response. 
The long-term improvement in the WBC count in the EP group could be ascribed to an improvement in the bloodproducing function of the bone marrow after PSE [26, 27]. The PLT count might have increased due to improvement in the hepatic production of thrombopoietin (TPO) after PSE, as well as due to the reduction in the splenic platelet pool and increase in the platelet survival time [28]. Interestingly, our study found that both the evaluated treatments, majorly the EP treatment, significantly improved the hemoglobin level and the RBC count for a long time. The hemoglobin level and the RBC count are relatively difficult to interpret, since they are influenced by transfusions, the confounding variance of variceal bleeding, and the effect of erythrocyte survival time. It is reported that RBC destruction occurs in the enlarged spleen at a rate of $0.5 \%-4.4 \%$ of the total RBC mass/day, with no evidence of immune hemolysis [29]. Increased hemoglobin level and RBC count in our study were attributed to the synergistic effects of PSE and endoscopic therapies. An increase in the erythrocyte survival time, decrease in the destruction of RBCs by monocytes and macrophages, activation of the bone marrow hematopoiesis after PSE, and decrease in the bleeding episodes after the EP treatment could all have led to the elevated hemoglobin levels and RBC count $[26,30]$.

Furthermore, our study demonstrated that the EP treatment is a potential therapeutic tool for improving therapeutic tool to improve liver function and coagulation function. The serum ALB concentration in the EP group increased by $9-13 \%$ compared to the baseline and was maintained at $>39 \mathrm{~g} / \mathrm{L}$ for 2 years. We found no significant changes in the other liver function parameters such as ALT, AST, AKP, and total bilirubin, which are more reflective of hepatocyte damage. As for the coagulation function indices, the EP group presented a remarkable decrease in PT and INR and a significant increase in PTA. The parameters that improved in the EP group included albumin, PT, and PTA, which are all indicators of the liver's ability to synthesize proteins. In cirrhosis patients, especially those in the decompensated stage, the hemostatic function is often disrupted, because most pro- and anticoagulant proteins are synthesized in the hepatic parenchymal cells. The improvements in PT, PTA, and INR in the EP group demonstrated that this treatment could not only improve the hepatic protein synthetic capabilities but could also maintain the coagulation and anticoagulation homeostasis.

The precise mechanisms of the EP treatment underlying the improvement in the liver function and coagulation function are currently unclear, although it is reported that they may be related to the hemodynamic alterations and changes in the host immunological factors [30-33]. We observed a decreased splenic venous flow and an increased flow of both hepatic artery and superior mesenteric artery under DSA after PSE. The decrease in the portal blood flow and a relative increase in the gastrointestinal-derived blood supply post-PSE may increase the supply of nutrients and cytokines to the liver. Research shows that an immunological mechanism may also be involved in the improvement in the liver function. An increased amount of PCNA-positive hepatocytes was observed in the post-PSE liver. These cells are reported to exhibit high proliferative activity, indicating that PSE may induce a liver regenerative response [27]. In addition, PSE may induce the activation of host immunity via complicated interactions between $\mathrm{T}$ cells and cytokines, and thereby improving liver function and coagulation function.

As for all research, the present study also had certain limitations. First, the follow-up period was short. Thus, a study with a long-term follow-up is needed to verify our findings and assess the long-term survival prognosis. Second, we did not measure the portal pressure and hemodynamics after the PSE. Nonetheless, based on our multiple positive results, the application of PSE combined with conventional endoscopic therapies could be included in the clinical guidelines for the secondary prophylaxis management of $\mathrm{VH}$. Third, the safety and efficiency of the EP treatment in cirrhotic patients with Child-Pugh score $\mathrm{C}$ was not fully assessed. Future studies with Child-Pugh score $\mathrm{C}$ patients are needed to verify this aspect.

In conclusion, the EP treatment is effective in reducing variceal bleeding and variceal recurrence. Moreover, it can chronically increase the peripheral blood cell count and improve the liver function, coagulation function, and the Child-Pugh score. Therefore, EP treatment proposed as an effective therapy superior to the conventional endoscopic therapies for the secondary prophylaxis of $\mathrm{VH}$ in cirrhosis patients with hypersplenism.

Supplementary Information The online version contains supplementary material available at https://doi.org/10.1007/s12072-021-10155-0.

Acknowledgements We are sincerely grateful to the physicians and all co-medical staff.

Author contributions YG and XL designed and administrated the study, gave critical comments of the manuscript equally. XS conducted the study, collected and interpreted the data, drafted the manuscript. $\mathrm{MW}, \mathrm{YC}, \mathrm{TZ}, \mathrm{XC}, \mathrm{AX}$ and $\mathrm{ZP}$ collected the date. AZ, TZ and BC interpreted the result and supervised the study. All authors had access to the study data and had reviewed and approved the final manuscript.

Funding There is no financial support of this study.

Data Availability All date generated or analyzed during this study are included in this published article.

\section{Compliance with ethical standards}

Conflict of interest Xin Sun, Anzhong Zhang, Tao Zhou, Minghui Wang, Yong Chen, Ting Zhou, Xiaoning Chen, Aiyuan Xiu, Zhi Peng, 
Baoquan Cheng, Xiaofeng Liu, Yanjing Gao declare that there is no conflict of interest.

Ethical approval All procedures followed were in accordance with the ethical standards of the responsible committee on human experimentation and with the Helsinki Declaration of 1975. Each institution approved the protocol, and the data of all patients were recorded in a standard form. All patients were informed of the aims of the study and signed the informed consent before participation.

Animal Research This article does not contain any studies with animal subjects.

Informed consent Informed consent for publication was obtained from all participants (Xin Sun, Anzhong Zhang, Tao Zhou, Minghui Wang, Yong Chen, Ting Zhou, Xiaoning Chen, Aiyuan Xiu, Zhi Peng, Baoquan Cheng, Xiaofeng Liu, Yanjing Gao).

Open Access This article is licensed under a Creative Commons Attribution 4.0 International License, which permits use, sharing, adaptation, distribution and reproduction in any medium or format, as long as you give appropriate credit to the original author(s) and the source, provide a link to the Creative Commons licence, and indicate if changes were made. The images or other third party material in this article are included in the article's Creative Commons licence, unless indicated otherwise in a credit line to the material. If material is not included in the article's Creative Commons licence and your intended use is not permitted by statutory regulation or exceeds the permitted use, you will need to obtain permission directly from the copyright holder. To view a copy of this licence, visit http://creativecommons.org/licenses/by/4.0/.

\section{References}

1. Garcia-Tsao G, Sanyal AJ, Grace ND, Carey W, Practice Guidelines Committee of the American Association for the Study of Liver D, Practice Parameters Committee of the American College of G. Prevention and management of gastroesophageal varices and variceal hemorrhage in cirrhosis. Hepatology. 2007;46:922-38. https://doi.org/10.1002/hep.21907.

2. Brunner F, Berzigotti A, Bosch J. Prevention and treatment of variceal haemorrhage in 2017. Liver Int. 2017;37(Suppl 1):10415. https://doi.org/10.1111/liv.13277.

3. Puente A, Hernández-Gea V, Graupera I, Roque M, Colomo A, Poca M, et al. Drugs plus ligation to prevent rebleeding in cirrhosis: an updated systematic review. Liver Int. 2014;34:823-33. https://doi.org/10.1111/liv.12452.

4. Bosch J, Garcia-Pagan JC. Prevention of variceal rebleeding. Lancet (Lond Engl). 2003;361:952-4. https://doi.org/10.1016/s0140 -6736(03)12778-x.

5. de Franchis R, Baveno VIF. Expanding consensus in portal hypertension: report of the Baveno VI consensus workshop: stratifying risk and individualizing care for portal hypertension. J Hepatol. 2015;63:743-52. https://doi.org/10.1016/j.jhep.2015.05.022.

6. Chen J, Zeng XQ, Ma LL, Huang XQ, Tseng YJ, Wang J, et al. Long-term efficacy of endoscopic ligation plus cyanoacrylate injection with or without sclerotherapy for variceal bleeding. J Dig Dis. 2016;17:252-9. https://doi.org/10.1111/1751-2980.12331.

7. Li L, Duan M, Chen W, Jiang A, Li X, Yang J, et al. The spleen in liver cirrhosis: revisiting an old enemy with novel targets. J Transl Med. 2017;15:111. https://doi.org/10.1186/s12967-017-1214-8.

8. Giannini E, Botta F, Borro P, Risso D, Romagnoli P, Fasoli A, et al. Platelet count/spleen diameter ratio: proposal and validation of a non-invasive parameter to predict the presence of oesophageal varices in patients with liver cirrhosis. Gut. 2003;52:1200-5. https ://doi.org/10.1136/gut.52.8.1200.

9. N'Kontchou G, Seror O, Bourcier V, Mohand D, Ajavon Y, Castera L, et al. Partial splenic embolization in patients with cirrhosis: efficacy, tolerance and long-term outcome in 32 patients. Eur J Gastroenterol Hepatol. 2005;17:179-84. https://doi. org/10.1097/00042737-200502000-00008.

10. Sangro B, Bilbao I, Herrero I, Corella C, Longo J, Beloqui O, et al. Partial splenic embolization for the treatment of hypersplenism in cirrhosis. Hepatology. 1993;18:309-14.

11. Lee CM, Leung TK, Wang HJ, Lee WH, Shen LK, Liu JD, et al. Evaluation of the effect of partial splenic embolization on platelet values for liver cirrhosis patients with thrombocytopenia. World J Gastroenterol. 2007;13:619-22. https://doi.org/10.3748/wjg.v13. i4.619.

12. Tajiri T, Yoshida H, Obara K, Onji M, Kage M, Kitano S, et al. General rules for recording endoscopic findings of esophagogastric varices (2nd edition). Digest Endosc. 2010;22:1-9. https://doi. org/10.1111/j.1443-1661.2009.00929.x.

13. Bosch J, García-Pagán JC. Prevention of variceal rebleeding. Lancet. 2003;361:952-4. https://doi.org/10.1016/S0140 $-6736(03) 12778-X$.

14. Lo G-H. Endoscopic treatments for portal hypertension. Hepatol Int. 2018;12:91-101. https://doi.org/10.1007/s12072-017-9828-8.

15. Huang HC, Haq O, Utsumi T, Sethasine S, Abraldes JG, Groszmann RJ, et al. Intestinal and plasma VEGF levels in cirrhosis: the role of portal pressure. J Cell Mol Med. 2012;16:1125-33. https ://doi.org/10.1111/j.1582-4934.2011.01399.x.

16. Iwakiri Y. Pathophysiology of portal hypertension. Clin Liver Dis. 2014;18:281-91. https://doi.org/10.1016/j.cld.2013.12.001.

17. Peck-Radosavljevic M. Thrombocytopenia in chronic liver disease. Liver Int. 2017;37:778-93. https://doi.org/10.1111/liv.13317

18. Ohmoto K, Yoshioka N, Tomiyama Y, Shibata N, Takesue M, Yoshida K, et al. Improved prognosis of cirrhosis patients with esophageal varices and thrombocytopenia treated by endoscopic variceal ligation plus partial splenic embolization. Dig Dis Sci. 2006;51:352-8. https://doi.org/10.1007/s10620-006-3137-8.

19. Duan X, Zhang K, Han X, Ren J, Xu M, Huang G, et al. Comparison of percutaneous transhepatic variceal embolization (PTVE) followed by partial splenic embolization versus PTVE alone for the treatment of acute esophagogastric variceal massive hemorrhage. J Vasc Interven Radiol: JVIR. 2014;25:1858-65. https:// doi.org/10.1016/j.jvir.2014.08.019.

20. Wan YM, Li YH, Xu ZY, Wu HM, Wu XN, Xu Y. Comparison of TIPS alone and combined with partial splenic embolization (PSE) for the management of variceal bleeding. Eur Radiol. 2019;29:5032-41. https://doi.org/10.1007/s00330-019-06046-6.

21. Helaly AZ, Al-Warraky MS, El-Azab GI, Kohla MA, Abdelaal EE. Portal and splanchnic hemodynamics after partial splenic embolization in cirrhotic patients with hypersplenism. APMIS. 2015;123:1032-9. https://doi.org/10.1111/apm.12470.

22. Hadduck TA, McWilliams JP. Partial splenic artery embolization in cirrhotic patients. World J Radiol. 2014;6:160-8. https://doi. org/10.4329/wjr.v6.i5.160.

23. Cai M, Huang W, Lin C, Li Z, Qian J, Huang M, et al. Partial splenic embolization for thrombocytopenia in liver cirrhosis: predictive factors for platelet increment and risk factors for major complications. Eur Radiol. 2016;26:370-80. https://doi. org/10.1007/s00330-015-3839-4.

24. Zhu K, Meng X, Qian J, Huang M, Li Z, Guan S, et al. Partial splenic embolization for hypersplenism in cirrhosis: a long-term outcome in 62 patients. Dig Liver Dis. 2009;41:411-6. https://doi. org/10.1016/j.dld.2008.10.005. 
25. Tajiri T, Onda M, Yoshida H, Mamada Y, Taniai N, Kumazaki T. Long-term hematological and biochemical effects of partial splenic embolization in hepatic cirrhosis. Hepatogastroenterology. 2002;49:1445-8.

26. Lu YF, Li XQ, Han XY, Gong XG, Chang SW. Peripheral blood cell variations in cirrhotic portal hypertension patients with hypersplenism. Asian Pacific J Trop Med. 2013;6:663-6. https://doi. org/10.1016/s1995-7645(13)60115-7.

27. Matsukiyo Y, Nagai H, Matsui T, Igarashi Y. Host immunological effects of partial splenic embolization in patients with liver cirrhosis. J Immunol Res. 2018;15(2018):1746391. https://doi. org/10.1155/2018/1746391.

28. Hidaka H, Kokubu S, Saigenji K, Isobe Y, Maeda T. Restoration of thrombopoietin production after partial splenic embolization leads to resolution of thrombocytopenia in liver cirrhosis. Hepatol Res. 2002;23:265- https://doi.org/10.1016/s1386-6346(02)00002 -5 .

29. Christensen BE. Quantitative determination of splenic red blood cell destruction in patients with splenomegaly. Scand J Haematol. 1975;14:295-302. https://doi.org/10.1111/j.1600-0609.1975. tb02430.x.

30. Koconis KG, Singh H, Soares G. Partial splenic embolization in the treatment of patients with portal hypertension: a review of the english language literature. J Vasc Intervent Radiol : JVIR. 2007;18:463-81. https://doi.org/10.1016/j.jvir.2006.12.734.

31. Hayashi H, Beppu T, Masuda T, Okabe H, Imai K, Hashimoto D, et al. Large splenic volume may be a useful predictor for partial splenic embolization-induced liver functional improvement in cirrhotic patients. J Hepato Bili Pancreat Sci. 2014;21:51-7. https:// doi.org/10.1002/jhbp.1.

32. Mukaiya M, Hirata K, Yamashiro K, Katsuramaki T, Kimura H, Denno R. Changes in portal hemodynamics and hepatic function after partial splenic embolization (PSE) and percutaneous transhepatic obliteration (PTO). Cancer Chemother Pharmacol. 1994;33(Suppl):S37-41. https://doi.org/10.1007/bf00686666.

33. Ishikawa T, Kubota T, Horigome R, Kimura N, Honda H, Iwanaga $\mathrm{A}$, et al. Concurrent partial splenic embolization with transcatheter arterial chemoembolization for hepatocellular carcinoma can maintain hepatic functional reserve. Hepatology Res. 2014;44:1056-61. https://doi.org/10.1111/hepr.12222.

Publisher's Note Springer Nature remains neutral with regard to jurisdictional claims in published maps and institutional affiliations. 